\title{
CPOL: Complex phase order likelihood as a similarity measure for MR-CT registration
}

\author{
Alexander Wong *, David A. Clausi, Paul Fieguth \\ Department of Systems Design Engineering, University of Waterloo, Waterloo, ON, Canada N2L 3G1
}

\section{A R T I C L E I N F O}

\section{Article history:}

Received 30 April 2009

Received in revised form 9 October 2009

Accepted 12 October 2009

Available online $\mathrm{xxxx}$

\section{Keywords:}

Image registration

Complex phase order

CT

MR

Likelihood

Statistics

\begin{abstract}
A B S T R A C T
A novel similarity measure for registering magnetic resonance (MR) and computed tomography (CT) images has been designed and built. MR-CT registration methods often rely on the statistical intensity relationship between the images. The proposed similarity measure instead depends on the statistical relationship between the complex phase order between the images. By utilizing the complex phase order likelihood (CPOL) as a similarity measure, structural relationships instead of intensity relationships are explicitly used. This approach can be advantageous for MR-CT registration, where the intensities of the CT imagery have highly complex and nonlinear relationships with the intensities of corresponding MR imagery but simpler linear structural relationships. This new similarity measure has been tested on real MR-CT 3D volumes and has been evaluated based on fiducial registration error to determine alignment accuracy. Quantitative results show that CPOL is capable of achieving comparable alignment accuracy when compared to normalized mutual information, while being more robust to imaging artifacts such as noise.
\end{abstract}

() 2009 Elsevier B.V. All rights reserved.

\section{Introduction}

The objective of image registration is to determine the alignment between images acquired under differing conditions, such as time and imaging modality. Image registration has proven to be a valuable tool in different medical applications ranging from computer-assisted surgery (Sadowski et al., 2002) to disease analysis (Walimbe et al., 2006). In particular, multimodal image registration has garnered significant research interest in the medical community given the wide variety of medical imaging technologies that capture different anatomical and functional information about the human body and the need to combine all this information in a meaningful manner.

A wide variety of algorithms has been proposed over the years to tackle the problem of multimodal image registration, with several surveys published summarizing research in the area (Maintz and Viergever, 1998; Hill et al., 2001; Pluim et al., 2003). A popular class of multimodal image registration methods are those based on intensity mutual information (Viola and Wells, 1997, 1999; Maes et al., 1997), which rely on the statistics of the intensity relationships between images to align the images. Methods based on intensity mutual information can be generally separated into those based on the maximization of local mutual information (Maintz et al., 1998; Likar and Pernus, 2001; Ardizzone et al., 2007), and

\footnotetext{
* Corresponding author.

E-mail address: a28wong@engmail.uwaterloo.ca (A. Wong).
}

those based on the maximization of global mutual information (Rueckert et al., 1999; Viola and Wells, 1997; Maes et al., 1997). One issue faced when utilizing intensity mutual information is that the intensity relationship between two different imaging modalities, such as MR and CT, can be highly complex and nonlinear. Given the under-constrained nature of intensity mutual information, where no functional correlation is assumed (Roche et al., 1998), the resulting convergence plane can become highly non-convex and difficult to optimize. Furthermore, intensity mutual information does not consider any notion of proximity, which can result in additional cost non-monotonicity and thus making it difficult to converge to the correct alignment (Roche et al., 1998). To address this issue, Mellor and Brady $(2004,2005)$ proposed the use of phase mutual information, which utilizes the local phase relationship between the images instead, which implicitly takes into account spatial information and has been shown to exhibit reduced relationship complexity and hence allow for better convergence to the correct alignment.

Another popular class of multimodal registration approaches are those based on intensity remapping (Roche et al., 1998, 2000; Guimond et al., 2001; Thirion, 1998; Andronache et al., 2008). Of particular popularity are those based on the correlation ratio (Roche et al., 1998; Roche et al., 2000), a regression-based metric that attempts to find the best functional fit between image intensities and maps the intensities of images to that of the reference image. This concept has been subsequently extended (Andronache et al., 2008), where the intensities of the images are mapped to a 
single intensity space, though not the same as that of the reference image. However, these assumptions are often not true (Orchard, 2008), particularly in situations characterized by image non-uniformities related to the acquisition device (Oghabian et al., 2003), as well as situations characterized by highly complex and nonlinear intensity relationships such as MR-CT registration.

The main contribution of this paper is a similarity measure based on complex phase order likelihood (CPOL) for registration of MR and CT imagery. The similarity measure aims to bypass the issues associated with utilizing highly complex and nonlinear intensity relationships by utilizing structural relationships instead. CPOL is designed to be robust to noise and image artifacts by accounting for them both in the complex phase order representation stage and the likelihood computation stage. While both the phase mutual information measure proposed by Mellor and Brady $(2004,2005)$ and the proposed CPOL measure utilize complex phase, there are significant differences between the two measures. The phase mutual information utilizes complex phase in a direct manner and implicitly accounts for spatial information and structural relationships by providing a illuminationinvariant representation. However, similar structural relationships in the direct complex phase representation can have very different values. On the other hand, CPOL utilizes the complex phase order across multiple scales and explicitly accounts for spatial information and structural relationships, where similar structural relationships in the complex phase order representation have very similar values. While less nonlinear than intensity relationships, the complex phase relationships between images such as those produced using MR and CT is significantly more nonlinear when compared to complex phase order relationships, which are largely linear. As such, the measure proposed by Mellor and Brady must rely on mutual information to model the complex phase relationships whereas CPOL can utilize a more constrained likelihood measure.

\section{The CPOL similarity measure}

The CPOL similarity measure for MR-CT registration can be described as follows. The complex phase for the images being registered is computed using a complex wavelet transform, as described in Section 2.1. Based on the complex phase, the complex phase order likelihood is computed, as described in Section 2.2. The algorithm for computing CPOL is provided in Section 2.3.

\subsection{Complex phase from complex wavelet decomposition}

Let $f=f(\underline{x})$ and $g=g(\underline{x})$ denote the images being aligned. The complex phase $\phi_{s, \theta}(\underline{x})$ at each scale $s$ and orientation $\theta$ is computed for each pixel in $f$ and $g$ as follows. First, multi-scale complex wavelet representations of $f$ and $g$, denoted as $F$ and $G$, are constructed using a complex wavelet transform such as the dual-tree complex wavelet transform (Selesnick et al., 2005) and Log-Gabor complex wavelet transform (Fischer et al., 2007), with each of the $\alpha$ scales and $\beta$ orientations. While the over-complete Log-Gabor complex wavelet transform proposed by Fischer et al. (2007) was used for our implementation of CPOL, where the complex wavelet representation at each scale is un-decimated, the dual-tree complex wavelet transform may also be used as well. For all tests, the Log-Gabor complex wavelet transform was performed over $\beta=6$ orientations $\left(0^{\circ}, 30^{\circ}, 60^{\circ}, 90^{\circ}, 120^{\circ}\right.$, and $\left.150^{\circ}\right)$ and $\alpha=4$ scales (wavelengths of $3,9,27$, and 81 pixels were used to maintain a bandwidth of two octaves).

In the transform domain, each point $\underline{x}$ in $F$ and $G$ is represented by a set of $\alpha \beta$ complex wavelet responses $\Upsilon_{s, \theta}(\underline{x})$, where

$\Upsilon_{s, \theta}(\underline{x})=A_{s, \theta}(\underline{x}) \exp \left[j \phi_{s, \theta}(\underline{x})\right]$, where $A_{s, \theta}(\underline{x})$ and $\phi_{s, \theta}(\underline{x})$ is the complex amplitude and phase, respectively. Computing $A_{s, \theta}(\underline{x})$ and $\phi_{s, \theta}(\underline{x})$ directly from Eq. (1) can lead to unstable results under the presence of image artifacts. To address this issue, a stable estimate of $A_{s, \theta}(\underline{x})$ and $\phi_{s, \theta}(\underline{x})$ can be obtained using an adaptive kernelized approach as

$A_{s, \theta}(\underline{x})=\sqrt{\left(f(\underline{x}) * K * R_{s, \theta}^{e}\right)^{2}+\left(f(\underline{x}) * K * R_{s, \theta}^{o}\right)^{2}}$,

and,

$\phi_{s, \theta}(\underline{x})=\tan ^{-1}\left(\frac{f(\underline{x}) * K * R_{s, \theta}^{e}}{f(\underline{x}) * K * R_{s, \theta}^{o}}\right)$,

where $R_{s, \theta}^{e}$ and $R_{s, \theta}^{o}$ are the even- and odd-symmetric Log-Gabor quadrature pairs, $*$ denotes a convolution, and $K$ is the adaptive kernel defined as,

$K(\mathbb{S})=\exp \left(-\frac{\sigma(\underline{x})^{2}(f(\underline{x})-f(\mathbb{S}))^{2}}{\xi \sigma_{b}^{4}}\right)$,

where $\mathbb{S}$ is a sampled set around $\underline{x}, \sigma_{b}$ is the standard deviation in the background regions of $f, \sigma(\underline{x})$ is the local standard deviation around $\underline{x}$, and $\xi=10$ was found to be effective and is used for all tests. What Eq. (4) does is adapt the degree of regularization based on the underlying value and statistical characteristics of $f$ to better preserve structural characteristics while achieving stability under the presence of image artifacts.

\subsection{Complex phase order likelihood}

Given the complex phase $\phi_{s, \theta}(\underline{x})$, the complex phase order $\rho(\underline{x})$, which can be defined as the degree of complex phase alignment across all scales, at each pixel must now be computed. Complex phase order is well suited for capturing structural characteristics of images explicitly (Morrone and Burr, 1988; Thomson, 1999; Kovesi, 2000) and is independent of intensity. Morrone and Owens (1987) proposed that the complex phase order $\rho(\underline{x})$ can be quantified based on the normalized weighted summation of cosineweighted complex phase deviations from the mean complex phase $\bar{\phi}_{\theta}(\underline{x})$ across all scales:

$\rho(\underline{x})=\frac{\sum_{\theta=1}^{\beta} \sum_{s=1}^{\alpha} A_{s, \theta}(\underline{x}) \Lambda(\underline{x})}{\sum_{\theta=1}^{\beta} \sum_{s=1}^{\alpha} A_{s, \theta}(\underline{x})}$,

where,

$\Lambda(\underline{x})=\cos \left(\phi_{s, \theta}(\underline{x})-\bar{\phi}_{\theta}(\underline{x})\right)$.

This approach to computing complex phase order has been further extended (Kovesi, 2000) to improve response sensitivity as well as robustness to noise using a hard thresholding approach. A limitation to this complex phase order formulation is that the use of a hard thresholding approach leads to the loss of potentially important information, resulting in a discontinuous representation that can affect the structural similarity across image modalities.

To address the hard thresholding limitation, we propose a modified complex phase order formulation that utilizes a bilateral softthresholding scheme. Motivated by work on wavelet denoising (Donoho, 1995), the use of a soft thresholding scheme allows for robustness to image noise while still providing a continuous measure for phase order. The bilateral soft thresholding scheme can be described as follows. Let us consider the numerator of Eq. (5), denoted as $\kappa(\underline{x})$, which corresponds to the summation of complex phase deviations at point $\underline{x}$,

$\kappa(\underline{\boldsymbol{x}})=\sum_{\theta=1}^{\beta} \sum_{s=1}^{\alpha} A_{s, \theta}(\underline{\boldsymbol{x}}) \Lambda(\underline{\boldsymbol{x}})$. 
Two general observations can be made with regards to image noise with respect to complex phase and amplitude relationships. First, in uniform regions within the image where there are no structural characteristics, the complex phase deviations across all scales from the mean phase should be zero. Therefore, non-zero summations of complex phase deviations in these uniform regions are entirely due to the influence of noise and should be suppressed accordingly. Second, the distribution of complex amplitude across multiple scales is generally narrow and skewed towards high frequencies, whereas for strong structural characteristics the complex amplitudes are generally high and distributed evenly across scales (Field and Brady, 1997). Therefore, we can perform soft thresholding by weighting the summation of complex phase deviations based on the non-zero summations in uniform regions as well as the distribution of complex amplitude across scales. The bilateral weighting function $W$ can therefore be expressed as a product of a weighting function $W_{v}(x)$ related to non-zero summations in uniform regions and a weighting function $W_{\tau}(\underline{x})$ related to the distribution of amplitude across scales:

$W(\underline{x})=W_{v}(\underline{x}) W_{\tau}(\underline{x})$,

where $W_{v}(\underline{\underline{x}})$ is governed by the median of the summations of phase deviations $\bar{\kappa}$ :

$W_{v}(\underline{x})=\frac{1}{1+\exp [\zeta(\bar{\kappa}-\kappa(\underline{x}))]}$

where $\zeta$ is the $W_{v}$ gain factor, and $W_{v}(\underline{x})$ is governed by an estimate of how evenly distributed complex amplitude is across scales (Kovesi, 2000),

$W_{\tau}(\underline{x})=\frac{1}{1+\exp \left[\epsilon\left(c-1+A_{\max , \theta}(\underline{x}) / \sum_{s=1}^{\alpha} A_{s, \theta}(\underline{x})\right)\right]}$.

where $c$ is the frequency where $W_{\tau}=0.5$ and $\epsilon$ is the $W_{\tau}$ gain factor. Based on testing, setting $c=0.4$ and $\epsilon=\zeta=10$ was found to be effective and is used for all tests. Eqs. (9) and (10) show that the penalty increases as the summation of complex phase deviations decreases to the range characterizing the uniform regions, as well as when the distribution of complex amplitude narrows respectively.

Integrating the bilateral soft thresholding scheme described in Eq. (8) into Eq. (5), the complex phase order $\rho(\underline{x})$ can be defined as

$\rho(\underline{x})=\frac{\sum_{\theta=1}^{\beta} \sum_{s=1}^{\alpha} W_{\theta}(\underline{x}) A_{s, \theta}(\underline{x}) \Lambda(\underline{x})}{\sum_{\theta=1}^{\beta} \sum_{s=1}^{\alpha} A_{s, \theta}(\underline{x})}$,

where $W_{\theta}(x)$ is the bilateral weighting function for orientation $\theta$. An example of an MR and CT image pair and a visualization of their corresponding complex phase order is shown in Fig. 1. While the MR and CT image volumes exhibit complex, nonlinear intensity relationships, the corresponding complex phase order exhibit simpler, linear structural relationships and as such can be compared in a more straightforward, constrained fashion that is more suitable for optimization. An example of the complex phase order of a MR image volume under $10 \%$ and $20 \%$ Rician noise is shown in Fig. 2. Despite the high noise levels, the resulting complex phase order remains robust to noise and effectively captures the structural characteristics of the MR image volume.

Given the complex phase order of $f$ and $g$, denoted as $\rho_{f}$ and $\rho_{g}$ respectively, the residual error between $\rho_{f}(\underline{x})$ and $\rho_{g}(\underline{x})$ can be defined as,

$\varepsilon(\underline{x})=\left(\rho_{f}(\underline{x})-\rho_{g}(\underline{x})\right)$.

Let the residual error $\varepsilon$ be a random variable following a probability distribution $p(\varepsilon ; f, g)$. The likelihood function $\mathscr{L}(f, g)$ can thus be defined as
$\mathscr{L}(f, g)=\prod_{x} p(\varepsilon(\underline{x}) ; f, g)$

Given that the error probability distribution $p(\varepsilon ; f, g)$ is generally unknown, choosing an error distribution model that provides a good characterization of the true error distribution is necessary. To better account for the presence of imaging noise and artifacts due to imaging modality differences (e.g., MR and CT), the error distribution model should be heavy-tailed. For CPOL, the following error distribution model based on the Geman-McClure function (Geman and McClure, 1987) is employed and can be defined as

$p(\varepsilon(\underline{x}) ; f, g)=\exp \left[-\frac{\varepsilon(\underline{x})^{2}}{\chi^{2}+\varepsilon(\underline{x})^{2}}\right]$.

Based on testing, setting $\chi=30$ was found to be effective and is used for all tests. A plot of the error distribution model based on the Geman-McClure function is shown in Fig. 3. The error distribution is characterized by a much heavier tail than the Gaussian distribution, thus taking the influence of outlier data such as noise and artifacts into account in the error distribution model. The final complex phase order likelihood $\mathscr{L}(f, g)$ can be defined as

$\mathscr{L}(f, g)=\prod_{\underline{x}} \exp \left[-\frac{\left(\rho_{f}(\underline{x})-\rho_{g}(\underline{x})\right)^{2}}{\chi^{2}+\left(\rho_{f}(\underline{x})-\rho_{g}(\underline{x})\right)^{2}}\right]$.

\subsection{Algorithm}

Based on the theory described in Section 2.1 and Section 2.2, the pseudo-code for computing CPOL between two images $f$ and $g$ is presented in Algorithm 1.

Algorithm 1. Complex phase order likelihood

1 Estimate complex amplitude $A_{f}, A_{g}(2)$ and phase $\phi_{f}, \phi_{g}$ (3) for $f$ and $g$.

2 Estimate complex phase order $\rho_{f}, \rho_{g}$ from $\left(A_{f}, \phi_{f}\right)$ and $\left(A_{g}, \phi_{g}\right)(11)$.

3 Compute complex phase order likelihood $\mathscr{L}$ between $\rho_{f}$ and $\rho_{g}(15)$.

\section{Experimental results}

The CPOL similarity measure was implemented in MATLAB and evaluated under 3D volume registration scenarios using 9 real patient MR-CT brain volume data sets from the Whole Brain Atlas (Johnson and Becker, 2008) (WBA). The MR volumes are acquired with an axial field of view of $24 \mathrm{~cm}$. The MR volumes are $256 \times 256 \times 23$ voxels, with a slice thickness of $5 \mathrm{~mm}$. The CT volumes are downsampled from an original resolution of $512 \times 512 \times 23$ voxels to the same voxel dimensions. A summary of each test data set is given below.

1. Test 1: MR/PD-CT, 63 year-old male, acute stroke.

2. Test 2: MR/T2-CT, 63 year-old female, acute stroke.

3. Test 3: MR/T2-CT, 45 year-old female, acute stroke.

4. Test 4: MR/T2-CT, 23 year-old female, hypertensive encephalopathy.

5. Test 5: MR/PD-CT, 42 year-old female, metastatic bronchogenic carcinoma.

6. Test 6: MR/PD-CT, 75 year-old male, meningioma.

7. Test 7: MR/T2-CT, 22 year-old male, sarcoma.

8. Test 8: MR/T2-CT, 55 year-old male, multiple embolic infarcts.

9. Test 9: MR/T2-CT, 71 year-old female, fatal stroke. 

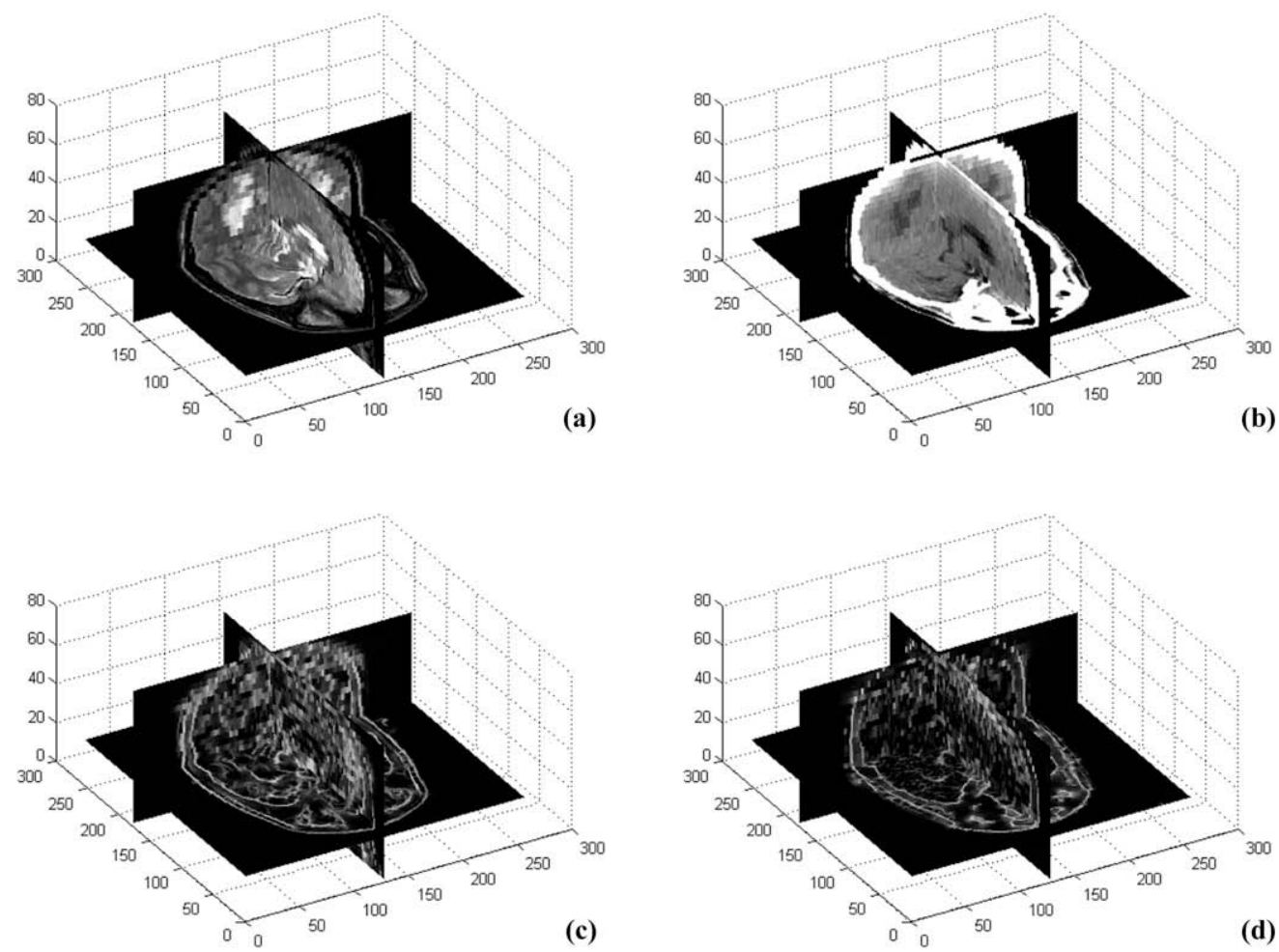

Fig. 1. A MR volume (a) and its complex phase order (c), and the corresponding CT volume (b) and its complex phase order (d). While the MR and CT image volumes (a)-(b) exhibit complex, nonlinear intensity relationships, the corresponding complex phase orders (c)-(d) exhibit simpler, linear structural relationships and as such can be compared in a more straightforward, constrained fashion that is more suitable for optimization.
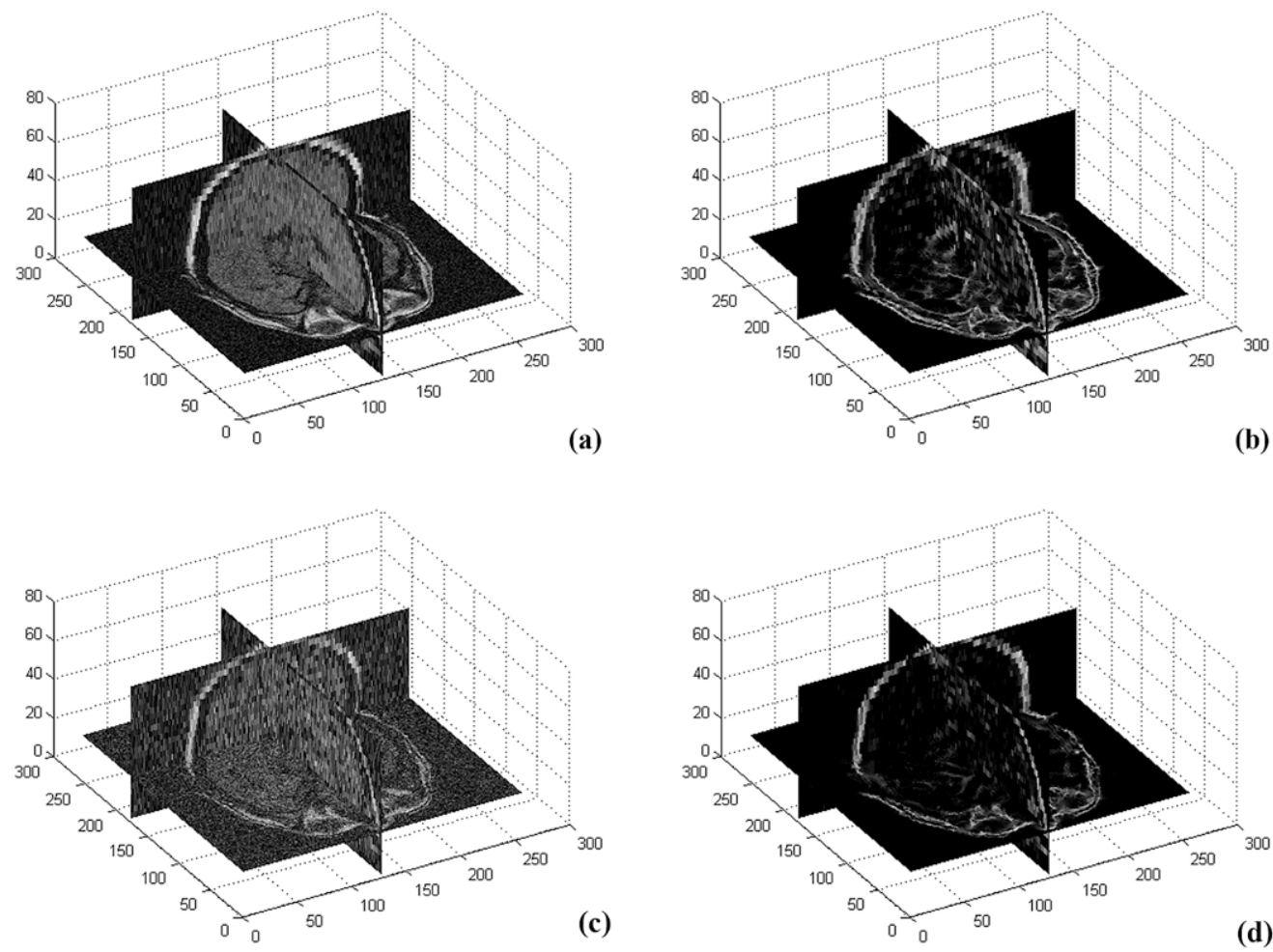

Fig. 2. A MR image volume under $10 \%$ Rician noise (a) and its complex phase order (b), and the same MR image volume under $20 \%$ Rician noise (c) and its complex phase order (d). Despite the high noise levels for both cases, the resulting complex phase orders (b)-(d) remain robust to noise and effectively captures the structural characteristics of the underlying MR image volume.

Please cite this article in press as: Wong, A., et al. CPOL: Complex phase order likelihood as a similarity measure for MR-CT registration. Med. Image Anal. (2009), doi:10.1016/j.media.2009.10.002 


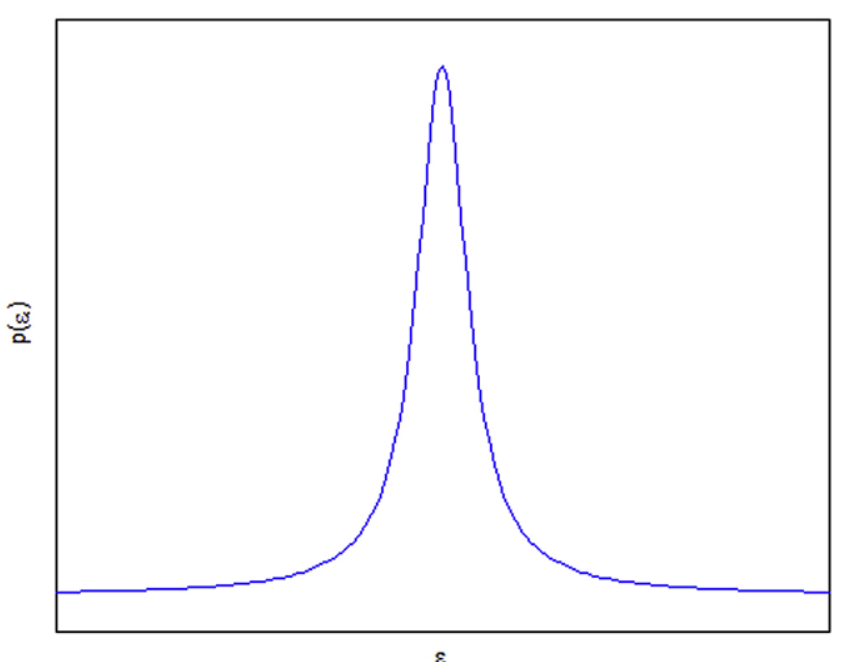

Fig. 3. Error distribution model based on the Geman-McClure function. The error distribution is characterized by a much heavier tail than the Gaussian distribution, thus taking the influence of outlier data such as noise and artifacts into account in the error distribution model.

For testing the similarity measures, the following 3D volume registration algorithm was used. The problem of registering images $f$ and $g$ is first formulated as an optimization problem, where the goal is to determine a transformation $T$ that aligns $f$ to $g$ such that the similarity measure $\Psi$ is maximized,

$\widehat{T}(\underline{x})=\arg \max _{T(\underline{x})}[\Psi(f(T(\underline{x})), g(\underline{x}))]$.

A sequential quadratic programming approach (Boggs and Tolle, 1995 ) is then employed to solve the problem in Eq. (16), where the estimated transformation $\widehat{T}$ at iteration $k$ can be defined as

$\widehat{T}_{k}=\widehat{T}_{k-1}+\gamma_{k-1} d_{k-1}$,

where $\gamma$ is a non-negative step size and $d$ is the step direction calculated by solving a quadratic subprogram involving $\Psi$ (Boggs and Tolle, 1995).

For evaluation purposes, the normalized mutual information (NMI) and the proposed CPOL similarity measures were tested. NMI was implemented using smoothed histograms with 100 intensity bins, with a Gaussian smoothing kernel of with $\sigma=7$ bins, as described in (Mellor and Brady, 2005). Trilinear interpolation was used in all experiments. All tests were performed on an Intel Core 2 Duo $1.67 \mathrm{GHz}$ PC with 2 GB of RAM.

\section{Experiment 1: registration accuracy}

The first set of tests studies the registration accuracy using NMI and CPOL under geometric distortion scenarios. Each test data set was distorted using 30 randomly generated affine transformations, based on the random perturbation of translation coefficients up to $\pm 30 \mathrm{~mm}$ and all other coefficients up to \pm 0.1 , resulting in a total of 270 test cases. Since the test image sets used were previously aligned, the gold-standard transformations are known for all 270 test cases. Registration accuracy for all methods is evaluated quantitatively based on the fiducial registration error (FRE), which in our case can be defined by the root-mean-square error of 60 fiducial points within the region of interest. The fiducial points were chosen randomly within the regions of interest in the test images as not to bias the tests towards any of the tested similarity measures.
Table 1

Fiducial registration errors (FRE) of NMI and CPOL for the nine data sets. A total of 30 random distortions is tested for each data set, for a total of 270 test cases.

\begin{tabular}{lll}
\hline Test set & FRE $(\mathrm{mm})$ & \\
\cline { 2 - 3 } & NMI & CPOL \\
\hline Test 1 & $3.74 \pm 0.56$ & $\mathbf{2 . 7 0} \pm 0.32$ \\
Test 2 & $3.91 \pm 0.50$ & $\mathbf{2 . 2 4} \pm 0.37$ \\
Test 3 & $\mathbf{2 . 3 6} \pm 0.63$ & $2.46 \pm 0.42$ \\
Test 4 & $3.64 \pm 0.49$ & $\mathbf{2 . 1 9} \pm 0.35$ \\
Test 5 & $3.81 \pm 0.30$ & $\mathbf{2 . 4 6} \pm 0.19$ \\
Test 6 & $3.71 \pm 0.47$ & $\mathbf{2 . 5 9} \pm 0.27$ \\
Test 7 & $2.90 \pm 0.53$ & $\mathbf{2 . 7 0} \pm 0.25$ \\
Test 8 & $3.35 \pm 0.55$ & $\mathbf{2 . 4 8} \pm 0.29$ \\
Test 9 & $3.97 \pm 0.61$ & $\mathbf{2 . 3 0} \pm 0.37$ \\
\hline
\end{tabular}
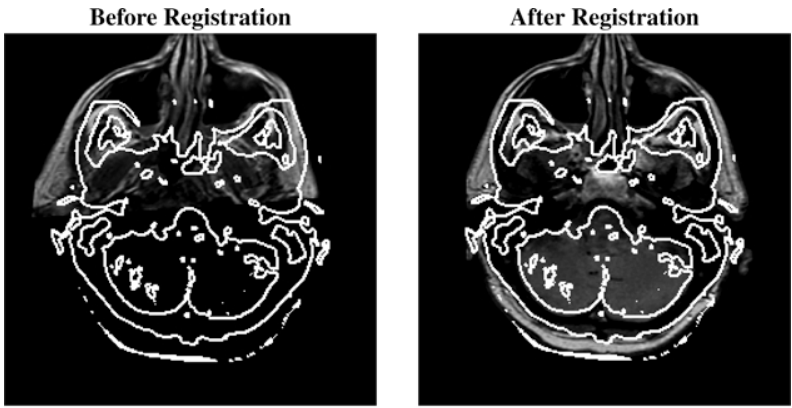

Slice 3
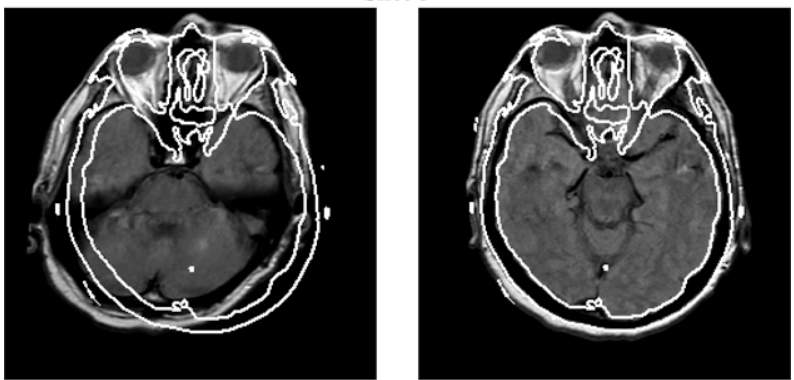

Slice 8
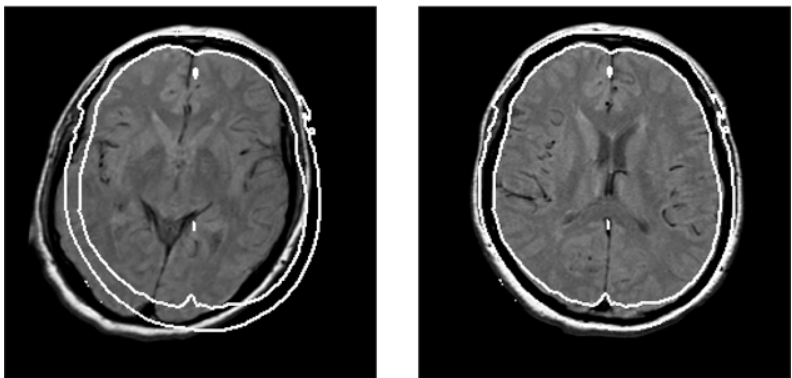

Slice 13
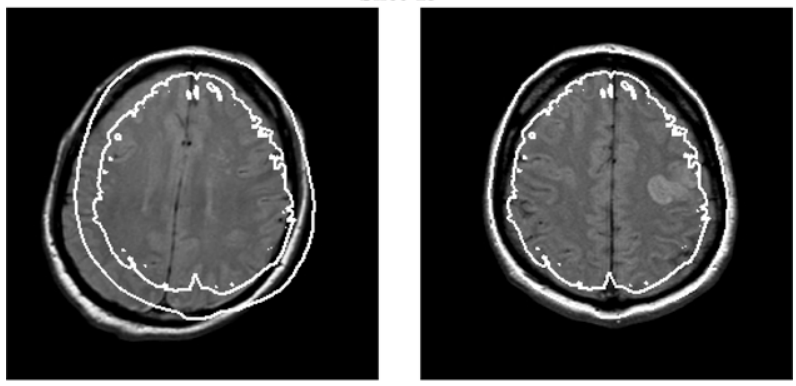

Slice 18

Fig. 4. Sample registration result from Test 1 using CPOL. For visualization, corresponding slices from the volumes before and after registration are shown. Contours extracted from the CT volume are overlayed on the MR volume to visualize the quality of registration. Visually, the MR volume warped based on CPOL appears well aligned with the CT volume. 


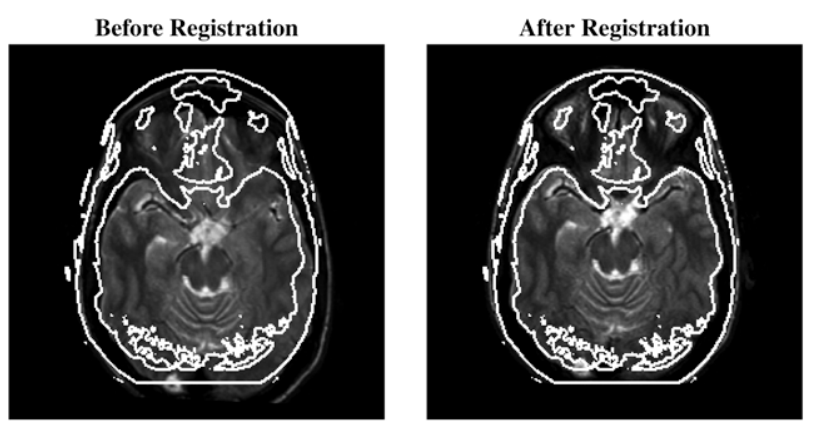

Slice 8

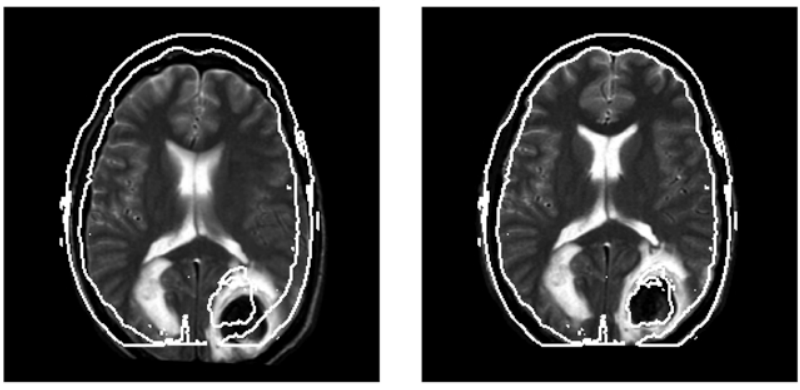

Slice 13

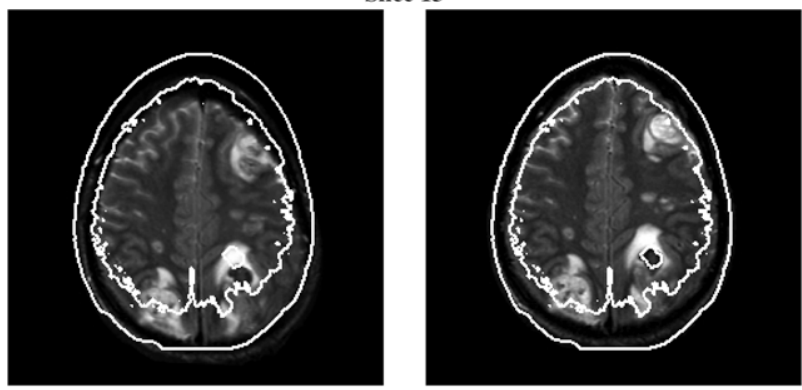

Slice 18

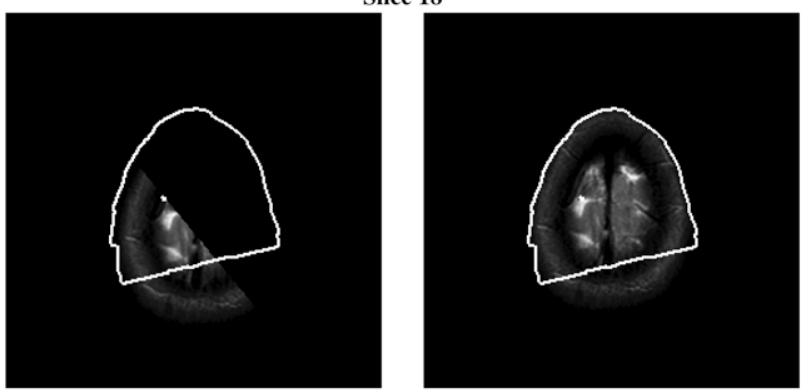

Slice 23

Fig. 5. Sample registration result from Test 7 using CPOL. For visualization, corresponding slices from the volumes before and after registration are shown. Contours extracted from the $\mathrm{CT}$ volume are overlayed on the MR volume to visualize the quality of registration. Visually, the MR volume warped based on CPOL appears well aligned with the CT volume.

The registration results for all nine test data sets, totalling 270 test cases, are summarized in Table 1. CPOL was capable of achieving noticeably lower or comparable FRE when compared to NMI for all test cases. One contributing factor to this difference in registration error when compared to NMI is that the intensity relationships between the tested MR and CT volume data sets are highly complex and nonlinear, making NMI highly non-convex and difficult to optimize. On the other hand, the structural relationships between the volume data sets is significantly more straightforward, making CPOL more straightforward to optimize. Sample registration results using the CPOL for Tests 1 and 7 are shown in Figs. 4 and 5, respectively. Visually, CPOL is capable of providing accurate registration results. These experimental results demonstrate the

\section{Table 2}

Fiducial registration errors (FRE) of NMI and CPOL for Test 1 under different Rician noise levels for MR volume. A total of 30 random distortions is tested for each noise level, for a total of 120 test cases.

\begin{tabular}{llllr}
\hline Rican noise & \multicolumn{4}{l}{ FRE $(\mathrm{mm})$} \\
\cline { 2 - 5 } & $5 \%$ & $10 \%$ & $15 \%$ & \multicolumn{1}{l}{$20 \%$} \\
\hline NMI & $4.02 \pm 0.76$ & $6.73 \pm 1.03$ & $9.71 \pm 1.64$ & $10.86 \pm 2.58$ \\
CPOL & $2.80 \pm 0.41$ & $2.76 \pm 0.52$ & $2.93 \pm 0.66$ & $3.57 \pm 0.95$ \\
\hline
\end{tabular}

effectiveness of the CPOL as a similarity measure for performing non-rigid multimodal image registration on CT and MR images.
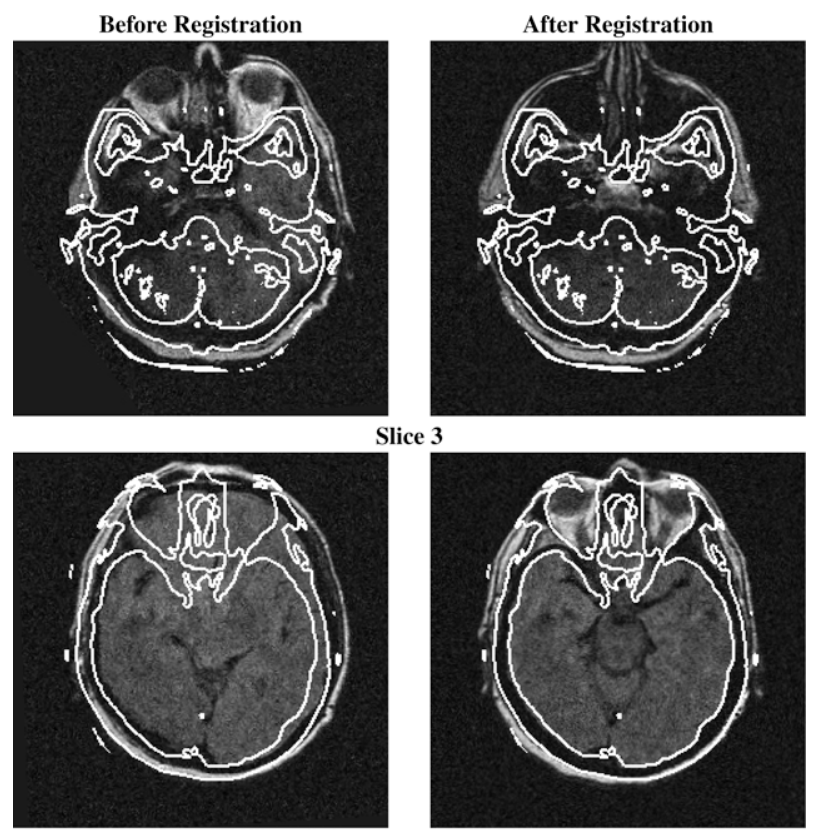

3

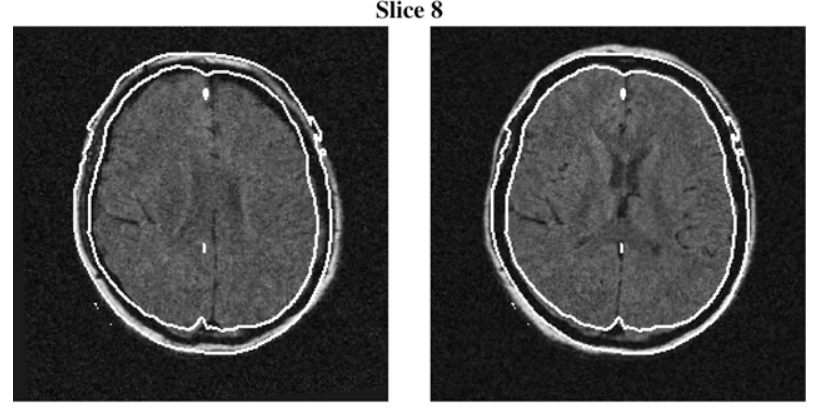

Slice 13

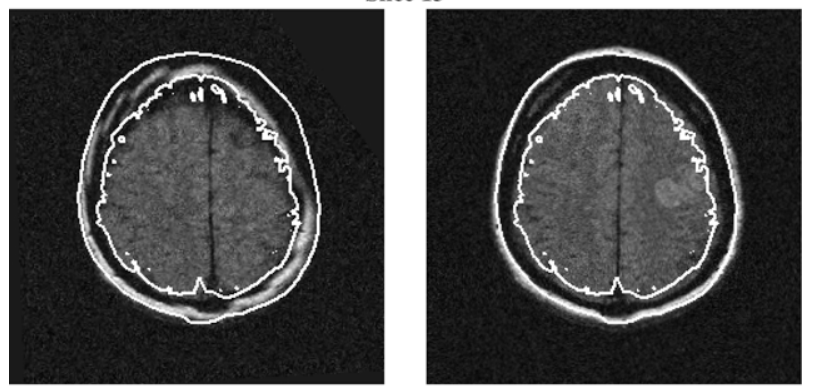

Slice 18

Fig. 6. Sample registration result from Test 1 using CPOL under $10 \%$ Rician noise For visualization, corresponding slices from the volumes before and after registration are shown. Contours extracted from the CT volume are overlayed on the MR volume to visualize the quality of registration. Visually, the MR volume warped based on CPOL appears well aligned with the CT volume despite noise. 


\section{Experiment 2: noise sensitivity}

An important consideration in the design of a similarity measure for image registration is that the measure should be robust to noise artifacts that can affect registration accuracy. To study the effect of noise on the similarity measures, the set of tests conducted in Section 4 for Test 1 were performed with the MR volumes contaminated by $5 \%, 10 \%, 15 \%$, and $20 \%$ simulated Rician noise, resulting in a total of 120 tests.

The registration results for the Test 1 under the various noise levels, totalling 120 test cases, are summarized in Table 2 . The
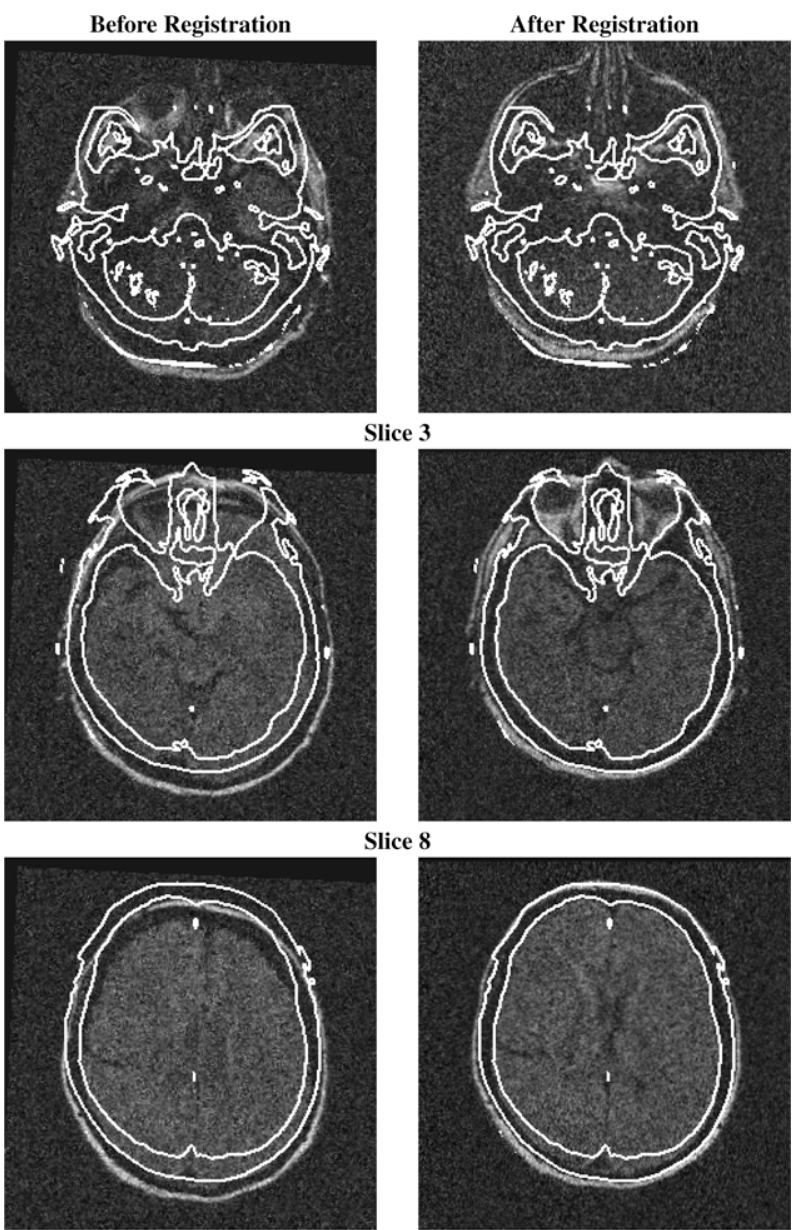

8
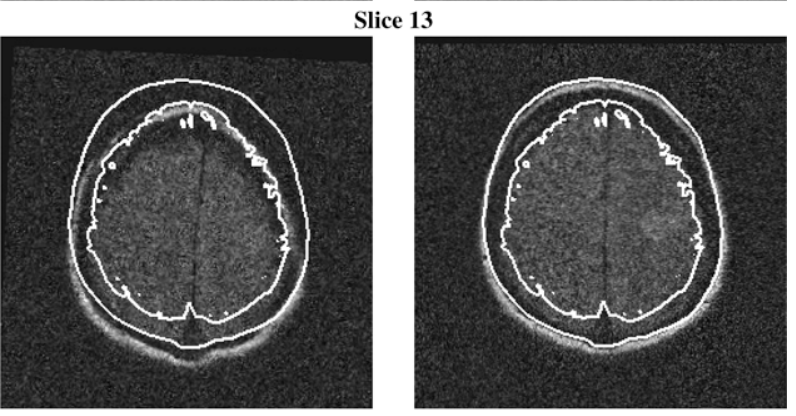

Slice 18

Fig. 7. Sample registration result from Test 1 using CPOL under $20 \%$ Rician noise. Contours extracted from the CT volume are overlayed on the MR volume to visualize the quality of registration. For visualization, corresponding slices from the volumes before and after registration are shown. Contours extracted from the CT volume are overlayed on the MR volume to visualize the quality of registration. Visually, the MR volume warped based on CPOL appears well aligned with the CT volume despite noise for the most part, with the exception of a $1.5 \mathrm{~mm}$ misalignment along the $y$-axis.
FRE achieved using CPOL remained largely consistent at all noise levels except $20 \%$ simulated Rician noise, where there is a noticeable increase in FRE. This increase in FRE can be contributed to the significant loss in structural characteristics due to the high level of noise. On the other hand, the FRE achieved using NMI rose significantly as noise levels increased. Sample registration results using the CPOL for $10 \%$ and $20 \%$ simulated Rician noise are shown in Figs. 6 and 7, respectively. Visually, CPOL is capable of providing accurate registration results for the most part, with the exception of a $1.5 \mathrm{~mm}$ misalignment along the $y$-axis for the $20 \%$ simulated Rician noise scenario. These experimental results demonstrate the robustness of CPOL to the presence of noise artifacts.

\section{Conclusions}

In this paper, we introduced a novel similarity measure for MRCT image registration. The complex phase order likelihood between the images was introduced for modality-invariant similarity measurement that is straightforward and robust to noise. Experimental results using real patient MR and CT volume data sets indicate that state-of-the-art registration accuracy can be achieved using CPOL when compared to NMI, particularly under high noise levels. Future work involves investigating more robust techniques for estimating complex phase order under the presence of imaging artifacts, as well as alternative distance measures that provide greater response to common characteristics between images acquired using different imaging modalities.

\section{Acknowledgements}

This research has been sponsored by the Natural Sciences and Engineering Research Council of Canada (NSERC) and Geomatics for Informed Decisions (GEOIDE).

\section{References}

Andronache, A., von Siebenthal, M., Szekely, G., Cattin, P., 2008. Non-rigid registration of multi-modal images using both mutual information and crosscorrelation. Medical Image Analysis 12, 3-15.

Ardizzone, E., Gambino, O., La Cascia, M., Lo Presti, L., Pirrone, R., 2007. Multi-modal non-rigid registration of medical images based on mutual information maximization. In: Proceedings of the International Conference on Image Analysis and Processing, pp. 743-750.

Boggs, P., Tolle, J., 1995. Sequential quadratic programming. Acta Numerica, 151.

Donoho, D., 1995. De-noising by soft-thresholding. IEEE Transactions on Information Theory 41 (3), 613-627.

Field, D., Brady, N., 1997. Visual sensitivity, blur and the sources of variability in the amplitude spectra of natural scenes. Vision Research 37 (23), 3367-3383.

Fischer, S., Sroubek, F., Perrinet, L., Redondo, R., Cristoba, G., 2007. Self-invertible 2D Log-Gabor wavelets. International Journal of Computer Vision 75 (2), 231-246.

Geman, S., McClure, D., 1987. Statistical methods for tomographic image reconstruction. Bulletin of the International Statistical Institute LII-4, 521.

Guimond, A., Roche, A., Ayache, N., Meunier, J., 2001. Three-dimensional multimodal brain warping using the demons algorithm and adaptive intensity corrections. IEEE Transactions on Medical Imaging 20 (1), 5869.

Hill, D., Batchelor, P., Holden, M., Hawkes, D., 2001. Medical image registration. Physics in Medicine and Biology 46, R1-R45.

Johnson, K., Becker, J., 2008. The whole brain atlas. <http://www.med.harvard.edu/ AANLIB/home.html>

Kovesi, P., 2000. Phase congruency: a low-level image invariant. Psychological Research 64 (2), 136-148.

Likar, B., Pernus, F., 2001. A hierarchical approach to elastic registration based on mutual information. Image and Vision Computing 19, 3344.

Maes, F., Collingnon, A., Vandermeulen, D., Marchal, G., Seutens, P., 1997. Multimodality image registration by maximization of mutual information. IEEE Transactions on Medical Imaging 16 (2), 187-198.

Maintz, J., Meijering, E., Viergever, M., 1998. General multimodal elastic registration based on mutual information. In: Proceedings of the SPIE Image Processing, vol. 3338, pp. 144-154.

Maintz, J., Viergever, M., 1998. A survey of medical image registration. Medical Image Analysis 2 (1), 136.

Mellor, M., Brady, 2004. M. Non-rigid multimodal image registration using local phase. In: Proceedings of the International Conference on Medical Image Computing and Computer Assisted Intervention, vol. 1, pp. 789-796. 
ARTICLE IN PRESS

8

A. Wong et al./Medical Image Analysis $x x x(2009) x x x-x x x$

Mellon, M., Brady, M., 2005. Phase mutual information as a similarity measure for registration. Medical Image Analysis 9, 330-343.

Morrone, M., Owens, R., 1987. Feature detection from local energy. Pattern Recognition Letters 6, 303-313.

Morrone, M., Burr, D., 1988. Feature detection in human vision: a phasedependent energy model. Proceedings of the Royal Society of London B $235,221-245$.

Oghabian, M., Mehdipour, S., Alum, N., 2003. The impact of RF inhomogeneity on MR image non-uniformity. In: Proceedings of the Image and Vision Computing, New Zealand.

Orchard, J., 2008. Multimodal image registration using floating regressor in the joint intensity scatter plot. Medical Image Analysis 12 (4), 385-396.

Pluim, J., Maintz, J., Viergever, M., 2003. Mutual-information-based registration of medical images: a survey. IEEE Transactions on Medical Imaging 22 (8), 9861004.

Roche, A., Malandain, G., Pennec, X., Ayache, N., 1998. The correlation ratio as a new similarity measure for multimodal image registration. In: Proceedings of the International Conference on Medical Image Computing and Computer Assisted Intervention, vol. 1496, pp. 1115-1124.

Roche, A., Pennec, X., Rudolph, M., Auer, D., Malandain, G., Ourselin, S., Auer, L., Ayache, N., 2000. Generalized correlation ratio for rigid registration of 3D ultrasound with MR images. In: Proceedings of the International Conference on
Medical Image Computing and Computer Assisted Intervention, vol. 1935, pp. 567-577.

Rueckert, D., Sonoda, L., Hayes, C., Hill, D., Leach, M., Hawkes, D., 1999. Nonrigid registration using freeform deformations: application to breast MR images. IEEE Transactions on Medical Imaging 18 (8), 712-721.

Sadowski, O., Yaniv, Z., Joskowicz, L., 2002. Comparative in-vitro study of contact and image-based rigid registration for computer-aided surgery. ComputerAided Surgery 7 (4), 223-236.

Selesnick, I., Baraniuk, R., Kingsbury, N., 2005. The dual-tree complex wavelet transform. IEEE Signal Processing Magazine 22 (6), 123-151.

Thirion, J., 1998. Image matching as a diffusion process: an analogy with Maxwell's demons. Medical Image Analysis 2 (3), 243260.

Thomson, M., 1999. Visual coding and the phase structure of natural scenes. Network: Computation in Neural Systems 10, 123132.

Viola, P., Wells, W., 1997. Alignment by maximization of mutual information. International Journal of Computer Vision 24 (2), 137-154.

Viola, P., Wells, W., 1999. An overlap invariant entropy measure of 3D medical image alignment. Pattern Recognition 32 (1), 71-86.

Walimbe, V., Dandekar, O., Mahmoud, F., Shekhar, R., 2006. Automated 3D elastic registration for improving tumor localization in whole-body PET-CT from combined scanner. In: Proceedings of the Annual International Conference of the IEEE Engineering in Medicine and Biology Society, vol. 1, pp. 2799-2802.

Please cite this article in press as: Wong, A., et al. CPOL: Complex phase order likelihood as a similarity measure for MR-CT registration. Med. Image Anal.

(2009), doi:10.1016/j.media.2009.10.002 